\title{
Síntese e propriedades fotoluminescentes do óxido de cério dopado com magnésio
}

\section{(Synthesis and photoluminescent properties of cerium oxide doped with magnesium)}

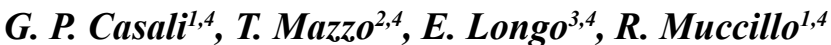 \\ ${ }^{I}$ Centro de Ciência e Tecnologia de Materiais, Instituto de Pesquisas Energéticas e Nucleares - IPEN, \\ S. Paulo, SP, Brazil \\ ${ }^{2}$ Departamento de Química,Universidade Federal de S. Carlos - UFSCar, S. Carlos, SP, Brazil \\ ${ }^{3}$ Instituto de Química, Universidade Estadual Paulista - UNESP, Araraquara, SP, Brazil \\ ${ }^{4}$ Centro de Desenvolvimento de Materiais Funcionais - CDMF \\ graziela@liec.ufscar.br
}

\begin{abstract}
Resumo
Neste trabalho são investigadas as propriedades ópticas de fotoluminescência do óxido de cério dopado com magnésio na forma de pós para analisar a influência dos sais precursores dos íons de interesse sobre a morfologia e as propriedades ópticas e para avaliar as propriedades ópticas com excitação em diferentes comprimentos de onda na região do visível e ultravioleta. Os pós foram obtidos pelo método dos precursores poliméricos. A caracterização estrutural dos materiais obtidos foi feita por difração de raios $\mathrm{X}$. A estrutura e a simetria dos materiais foram estudadas por espectrometria de espalhamento Raman. As curvas de transmissão foram avaliadas por espectroscopia eletrônica na região UV-Vis. As propriedades ópticas foram avaliadas por espectroscopia de fotoluminescência e a microestrutura por microscopia eletrônica de varredura.

Palavras-chave: cério, fotoluminescência, método dos precursores poliméricos.
\end{abstract}

\begin{abstract}
The optical properties of the photoluminescence of cerium oxide doped with magnesium in the form of powders were investigated. The influence of the precursor salts of the ions of interest on the morphology and optical properties and the optical properties with excitation at different wavelengths in the visible and ultraviolet region were evaluated. The powders were synthesized by the polymeric precursor method. The structural characterization of the materials was carried out by X-ray diffraction. The symmetry of the structure was studied by Raman spectrometry. The optical transmission curves were evaluated by electron spectroscopy in the UV-Vis region. The optical properties were evaluated by photoluminescence spectroscopy and the microstructure by scanning electron microscopy.
\end{abstract}

Keywords: cerium, photoluminescence, polymeric precursor.

\section{INTRODUÇÃO}

$\mathrm{O}$ óxido de cério $\left(\mathrm{CeO}_{2}\right)$ apresenta estrutura cúbica tipo fluorita e é considerado, comparativamente aos de terrasraras como o mais importante, tendo sido extensivamente pesquisado produzindo-se muitas patentes de aplicações [1]. O óxido de cério é de grande importância por suas propriedades ópticas, alta estabilidade térmica, condutividade elétrica e difusividade, e a capacidade para armazenar e liberar oxigénio. Por estas propriedades, o $\mathrm{CeO}_{2}$ pode ser utilizado como abrasivo [2], pigmento [3], material de catálise [4], eletrólito sólido de condução de íons oxigênio [5], e sensor de oxigênio [6]. Nos últimos anos foram realizadas algumas tentativas para a preparação de óxido de cério dopado com íons terras raras por várias técnicas, tais como pirólise [7], síntese solvotérmica [8,9], síntese sonoquímica [10], coprecipitação de dois estágios $[11,12]$, processo sol-gel $[13,14]$, reações de estado sólido [15], irradiação por micro-ondas [16], etc. [9-12, 15-19].

O desenvolvimento de materiais semicondutores com atividade em propriedades ópticas como fotoluminescência, eletroluminescência ou óptica não linear pode levar ao desenvolvimento de novos dispositivos óptico-eletrônicos com desempenho superior. A luminescência é o caso geral no qual um fóton de maior energia é absorvido e um fóton de menor energia é emitido, mas esse termo geralmente vem acompanhado de um prefixo que está relacionado à fonte de energia excitante. $\mathrm{O}$ fenômeno luminescente pode ser classificado de acordo com a fonte excitante de energia. A fonte excitante de energia da fotoluminescência é a radiação eletromagnética [20].

Um aumento no interesse por materiais que apresentam 
propriedade fotoluminescente (FL) pode ser notado após ser reportada a emissão FL na região do visível em silício poroso à temperatura ambiente [21]. Esse trabalho é um marco no estudo da emissão FL em materiais, uma vez que anteriormente os estudos de emissão FL eram feitos a baixas temperaturas. O mais relevante nesse trabalho é o fato de terse observado emissão FL na região do visível à temperatura ambiente, pois favorece as aplicações tecnológicas. Em particular, nesse trabalho, o autor gerou defeitos estruturais em silício, tornando-o poroso, fazendo com que o material, que quando ordenado não apresentava emissão FL na região do visível à temperatura ambiente, passasse a apresentar essa emissão.

A fotoluminescência da céria foi reportada. Foi observado que a fotoluminescência em $\sim 400 \mathrm{x} \mathrm{nm}(3,10$ eV) em filmes finos de $\mathrm{CeO}_{2}$ depositados em substrato de Si (111) por ablação de laser pulsado, o que foi atribuído à $\mathrm{Ce}_{6} \mathrm{O}_{11}$ [22]. Foi também observada uma emissão azul forte, com um pico a $425 \mathrm{~nm}(2,92 \mathrm{eV})$ em nanopartículas $\mathrm{CeO}_{2}$ com tamanho médio de $1,8 \mathrm{~nm}$, preparadas pelo método hidrotérmico. Picos de emissão de luz violeta/azul em $405,389,382$, e $368 \mathrm{~nm}$ sob diferentes concentrações de nanopartículas de $\mathrm{CeO}_{2}$ diluídos em água bidestilada foram também reportadas [23]. A dependência do deslocamento do pico azul da fotoluminescência com a concentração de partículas de $\mathrm{CeO}_{2}$ também foi observada para as nanopartículas de $\mathrm{CeO}_{2}$ sintetizadas pela rota de micelas inversa [24]. Este fenômeno foi explicado pela transição de carga a partir da banda 4f para a banda de valência do $\mathrm{CeO}_{2}$ em ambas as nanopartículas e filmes finos [25, 26]. Nota-se também que nas amostras de $\mathrm{CeO}_{2}$ as emissões azul e azulesverdeadas fracas são possivelmente devido a defeitos de superfície nas nanopartículas de $\mathrm{CeO}_{2}$, e a baixa intensidade da emissão verde pode ser devida à baixa densidade de vacâncias de oxigênio na amostra de $\mathrm{CeO}_{2}$ [27]. A banda centrada em $406 \mathrm{~nm}(3,06 \mathrm{eV})$ na amostra de $\mathrm{CeO}_{2}$ originase do defeito existente entre o estado $4 \mathrm{f} \mathrm{Ce} \mathrm{e} 2 \mathrm{p} \mathrm{O}$ da banda de valência [22]. Estes defeitos possivelmente atuam como centros de recombinação radiativa para elétron inicialmente excitados da banda de valência para a banda $4 \mathrm{f}$ do $\mathrm{CeO}_{2}$ [25]. Um estudo das nanopartículas de $\mathrm{CeO}_{2}$ exibiu uma notável fotoluminescência no ultravioleta próximo, o que é atribuído principalmente ao estado de defeito abundante entre as bandas $4 \mathrm{f} \mathrm{Ce} \mathrm{e} 2 \mathrm{p} \mathrm{O}$, melhor cristalinidade e maior concentração de íons $\mathrm{Ce}^{3+}$ nas nanopartículas. $\mathrm{O}$ mecanismo de síntese possível de nanoestruturas de $\mathrm{CeO}_{2}$ com morfologia controlada foi discutido em termos da influência de cadeias de hidrocarbonetos, de constante dielétrica, e da viscosidade na solução em sistema de reação termodinâmica e cinética de nucleação [28].

A propriedade óptica de fotoluminescência é dependente do grau de organização estrutural, do método de preparação e das condições de tratamento térmico. Portanto, é importante compreender a influência de diferentes métodos de síntese no grau da organização estrutural e nas propriedades ópticas. Partindo deste pressuposto, relataremos a síntese de pós de $\mathrm{CeO}_{2}$ dopado com magnésio pelo método dos precursores poliméricos (MPP) e tratados termicamente a diferentes temperaturas.

\section{EXPERIMENTAL}

Inicialmente foi preparado o citrato metálico, sendo o ácido cítrico dissolvido em água destilada a $75{ }^{\circ} \mathrm{C}$ sob constante agitação. Em seguida, foi adicionado o nitrato de cério III a esta solução aquosa com o ácido cítrico. A relação estequiométrica foi de $1 \mathrm{~mol}$ de cério para 3 de ácido cítrico para garantir a completa quelação dos cátions pelo ácido. Este sistema foi mantido a $90{ }^{\circ} \mathrm{C}$ sob agitação constante até que se obteve uma solução transparente. A porcentagem em massa de cério na solução foi determinada por gravimetria. Para isso, foram calcinadas quantidades em massa previamente pesadas da solução em cadinhos de alumina a $950{ }^{\circ} \mathrm{C}$ por $2 \mathrm{~h}$. Em seguida, foram adicionados à solução de citrato de cério obtida os cátions de interesse para exercer a função de modificador de rede na estrutura do material a ser obtido, com íon magnésio utilizando o sal nitrato de magnésio e, em seguida, um polihidroxialcool, etileno glicol. A adição desse último reagente promove a poliesterificação e a formação da resina. Cada resina foi pré-calcinada a $200^{\circ} \mathrm{C}$ por $5 \mathrm{~h}$ para que ocorresse o início da pirólise do poliéster. $\mathrm{O}$ material obtido foi desaglomerado com almofariz e pistilo, obtendo-se o pó. Em seguida, esses pós foram tratados termicamente em forno convencional em 3 etapas: a) elevação da temperatura a 320 ${ }^{\circ} \mathrm{C}$ com taxa de aquecimento de $10^{\circ} \mathrm{C} /$ min e permanência de 60 min para a eliminação de orgânicos, b) para a posterior cristalização, a temperatura de tratamento foi elevada para $500,600,700$ e $800^{\circ} \mathrm{C}$ com taxa de aquecimento de $10^{\circ} \mathrm{C} / \mathrm{min}$ por $120 \mathrm{~min}$, e (c) resfriamento até a temperatura ambiente com taxa de $10{ }^{\circ} \mathrm{C} / \mathrm{min}$.

\section{RESULTADOS E DISCUSSÃO}

A Fig. 1 ilustra o difratograma de raios $\mathrm{X}$ obtido para as amostras de $\mathrm{CeO}_{2}$ dopadas com magnésio tratadas termicamente de 500 a $800^{\circ} \mathrm{C}$. A cristalização da fase $\mathrm{CeO}_{2}$ se inicia a $500{ }^{\circ} \mathrm{C}$, na qual se observa os picos de difração, indicando que acima desta temperatura as amostras estão organizadas a longa distância. Todos os picos de difração podem ser indexados como uma estrutura cúbica tipo fluorita, segundo o arquivo JCPDF 09-0418.

Na Fig. 2 pode-se observar que a uma mesma temperatura a amostra dopada com $2 \%$ de magnésio apresentou maior cristalinidade.

A espectroscopia micro-Raman é uma técnica utilizada neste trabalho com a finalidade de avaliar o grau de organização estrutural dos pós de $\mathrm{CeO}_{2}$ dopado com magnésio a curta distância. A Fig. 3 ilustra o espectro microRaman destes pós dopados com 1, 2 e 5\% de magnésio, tratados termicamente a $800{ }^{\circ} \mathrm{C}$. As amostras apresentam um modo Raman ativo, o que indica que está ocorrendo uma organização estrutural a curta distancia. Este modo já foi reportado [29, 30], caracterizando uma estrutura ordenada a curta distância. Sabe-se que a estrutura fluorita $\mathrm{CeO}_{2}$ tem 

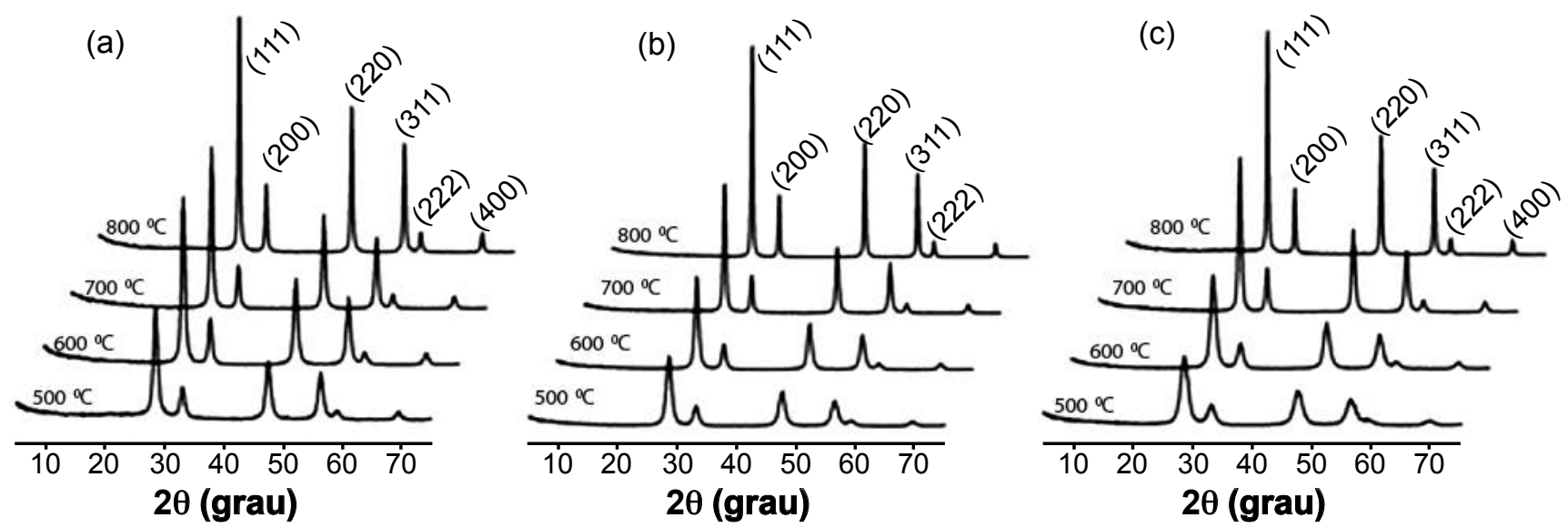

Figura 1: Difratograma de raios $\mathrm{X}$ dos pós de $\mathrm{CeO}_{2}$ dopados com (a) $1 \%$, (b) $2 \%$ e (c) $5 \%$ de magnésio, obtidos pelo método dos precursores poliméricos.

[Figure 1: X-ray diffraction patterns of $\mathrm{CeO}_{2}$ powders doped with (a) $1 \%$ (b) 2\% and (c) 5\% magnesium, obtained by the polymeric precursor method.]

apenas um único modo Raman permitido, com freqüência $465 \mathrm{~cm}^{-1}$, que pode ser assumido como modo simétrico "breathing" F2g dos átomos de $\mathrm{O}$ em torno de cada cátion.

Nos modelos que buscam explicar a emissão fotoluminescente em materiais, o principal evento ocorre após o processo de excitação. Um modelo para explicar a emissão fotoluminescência em materiais desordenados, baseado em eventos anteriores ao processo de excitação, chamado "modelo de banda larga" foi proposto, fundamentado em resultados experimentais e teóricos [31, 32]. Os espectros foram obtidos à temperatura ambiente com comprimento de onda de excitação fixo em $350,7 \mathrm{~nm}$ à temperatura ambiente. A banda larga de emissão fotoluminescência observada nos espectros em todas as amostras é característica do comportamento de um processo multifônico, ou seja, os

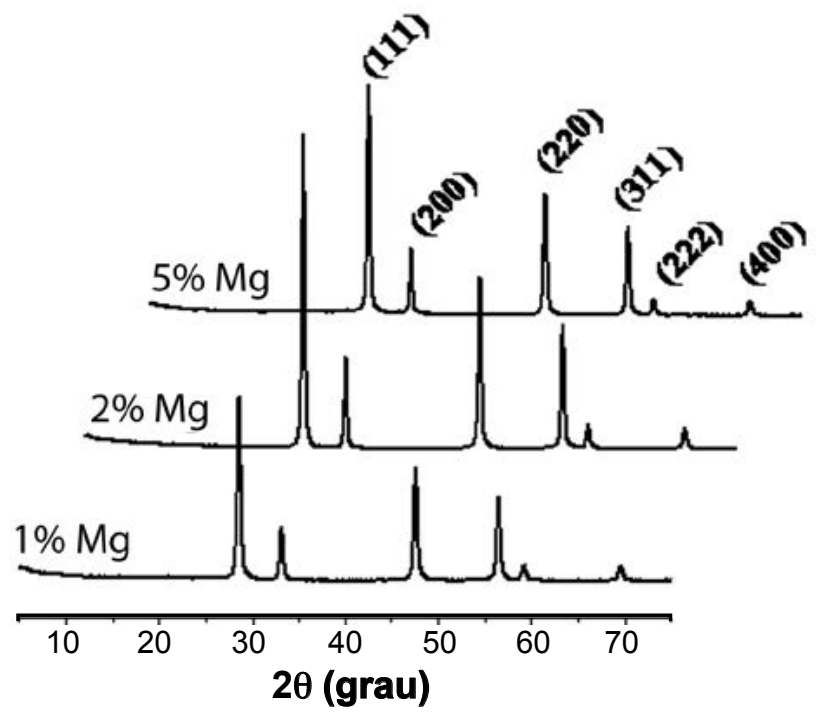

Figura 2: Difratogramas de raios $\mathrm{X}$ dos pós de $\mathrm{CeO}_{2}$ dopados com magnésio, obtidos pelo método dos precursores poliméricos a $800{ }^{\circ} \mathrm{C}$.

[Figure 2: X-ray diffraction patterns of the $\mathrm{CeO}_{2}$ powders doped with magnesium, obtained by the polymeric precursor method to $\left.800{ }^{\circ} \mathrm{C}.\right]$ sistemas apresentam um processo de relaxação com várias etapas e estados localizados na banda de "gap" dos materiais que surgem devido aos defeitos ou distorções presentes na estrutura.

As curvas de fotoluminescência das amostras de $\mathrm{CeO}_{2}$ dopadas com 1, 2 e $5 \%$ de magnésio e sintetizadas em diferentes temperaturas estão ilustradas na Fig. 4.

O perfil das bandas é de um espectro de banda larga em todo o espectro da luz visível, de 350 a $800 \mathrm{~nm}$. É possível observar que as amostras obtidas em $800{ }^{\circ} \mathrm{C}$ apresentam maior intensidade fotoluminescente. Portanto, o aumento da temperatura de tratamento térmico favoreceu uma maior luminescência. Com o intuito de verificar o efeito do dopante magnésio na propriedade fotoluminescente do $\mathrm{CeO}_{2}$, foram comparados os espectros das amostras obtidas em $800{ }^{\circ} \mathrm{C}$, pois foram os que apresentaram uma maior intensidade fotoluminescente.

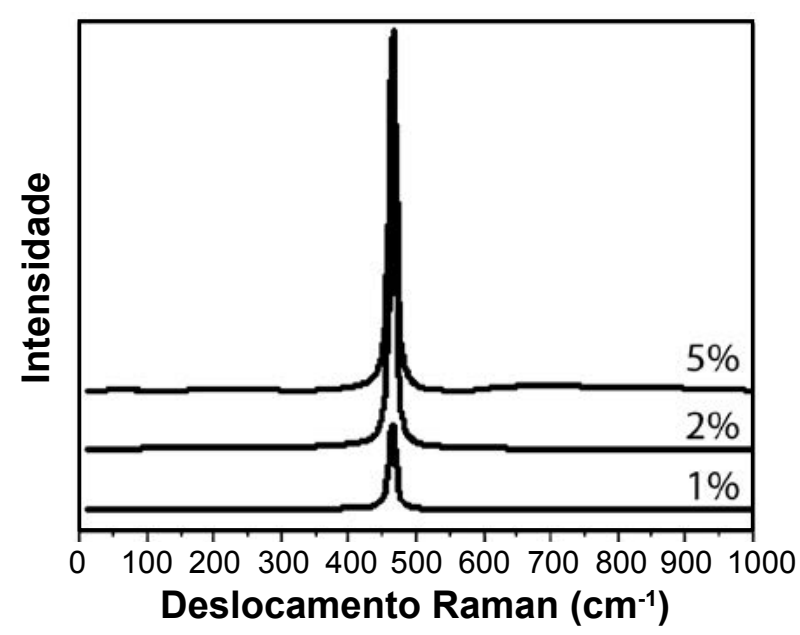

Figura 3: Espectros micro-Raman dos pós de $\mathrm{CeO}_{2}$ dopados com $1 \%, 2 \%$ e $5 \%$ de magnésio, tratados termicamente a $800{ }^{\circ} \mathrm{C}$.

[Figure 3: Micro-Raman spectra of $\mathrm{CeO}_{2}$ powders doped with 1\%, $2 \%$ and $5 \%$ magnesium, thermally treated at $800{ }^{\circ} \mathrm{C}$.] 

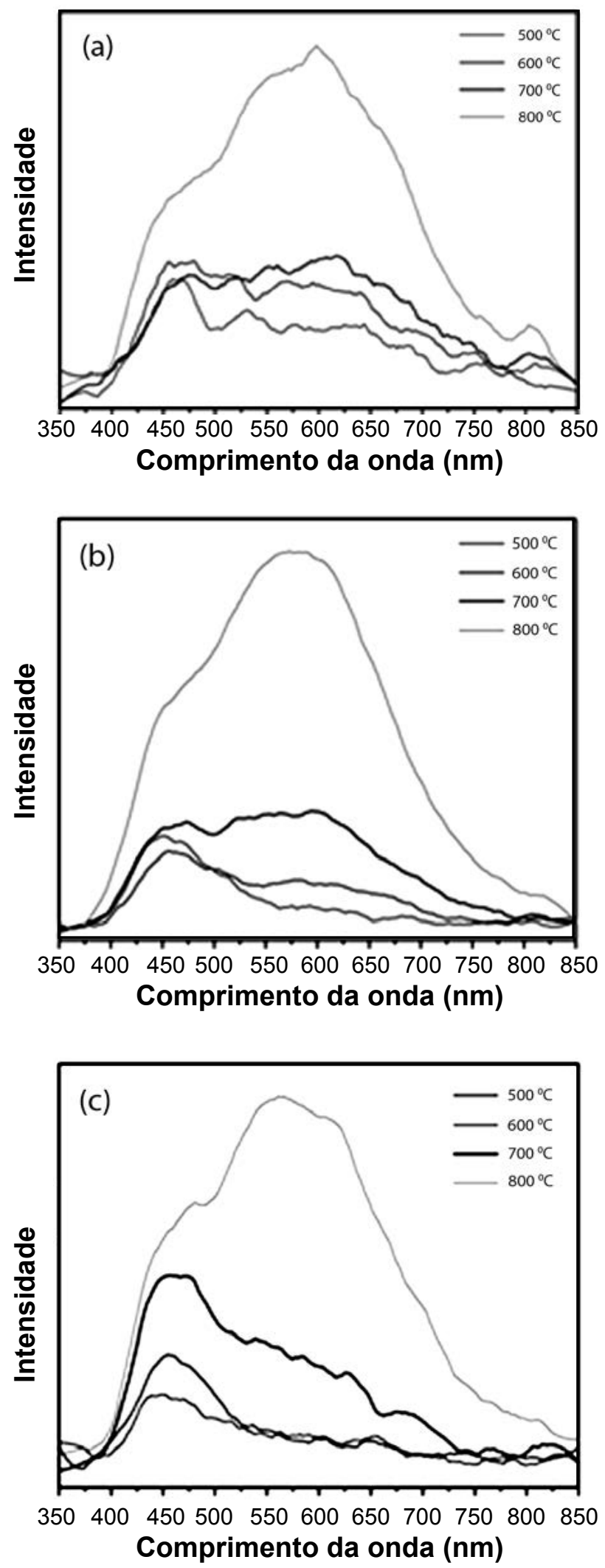

Figura 4: Espectros de fotoluminescência das amostras de $\mathrm{CeO}_{2}$ dopadas com (a) $1 \%$, (b) $2 \%$ e (c) $5 \%$ de magnésio e sintetizadas em diferentes temperaturas.

[Figure 4: Photoluminescence spectra of doped $\mathrm{CeO}_{2}:(a): 1 \%$, (b) $2 \%$ and (c) 5\% magnesium, synthesized at different temperatures.]
A Fig. 5 ilustra os espectros das amostras de $\mathrm{CeO}_{2}$ dopadas com 1,2 e $5 \%$ de magnésio obtidas a $800{ }^{\circ} \mathrm{C}$.

Observou-se a máxima intensidade de emissão fotoluminescência na amostra de $\mathrm{CeO}_{2}$ dopada com $2 \%$ de magnésio. A diminuição da intensidade de emissão para a amostra $\mathrm{CeO}_{2}$ dopada com $5 \%$ de magnésio pode ser atribuída ao "quenching" (supressão) de concentração. Ou seja, quando se introduz $5 \mathrm{~mol} \%$ de íon magnésio na matriz de $\mathrm{CeO}_{2}$, parte da energia absorvida quando essa amostra é excitada pode ser perdida por transferência de energia não-radiativa devido às interações íon-íon ou íon-vacâncias (defeitos gerados pela inserção do dopante na matriz). Por esse motivo, a luminescência é suprimida.

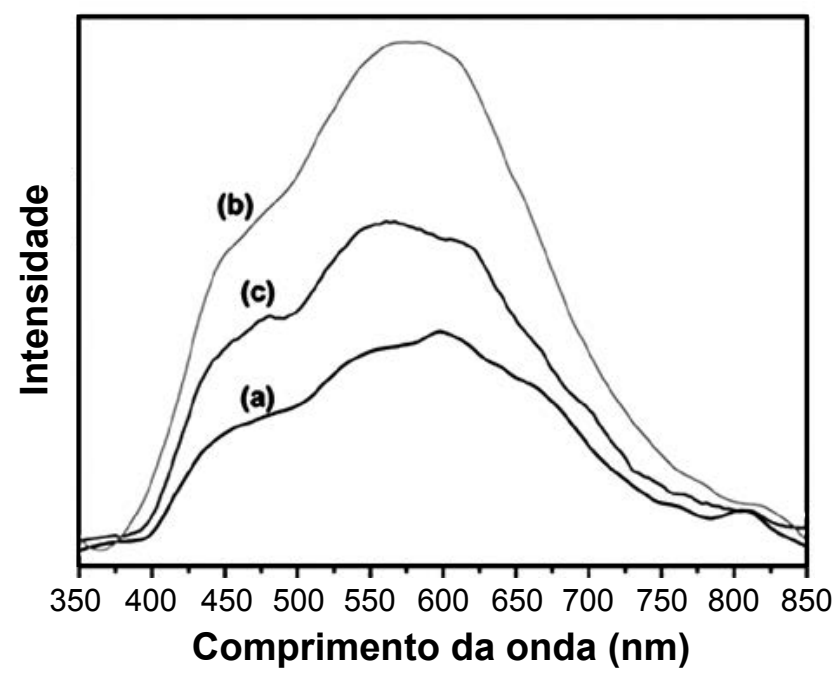

Figura 5: Espectros de fotoluminescência das amostras de $\mathrm{CeO}_{2}$ dopadas com (a) $1 \%$, (b) $2 \%$ e (c) $5 \%$ de magnésio, obtidas a $800{ }^{\circ} \mathrm{C}$. [Figure 5: Photoluminescence spectra of $\mathrm{CeO}_{2}$ doped with (a) $1 \%$, (b) $2 \%$ and (c) $5 \%$ magnesiumat $800{ }^{\circ} \mathrm{C}$.]

É conhecido que a fotoluminescência é dependente do grau de ordem-desordem do material; assim, essa propriedade acompanha a evolução estrutural do material. Foi observado por difração de raios $\mathrm{X}$ que a amostra $\mathrm{CeO}_{2}$ dopada com $2 \%$ de magnésio apresenta uma maior cristalinidade, ou seja, organização estrutural a longa distancia devido à maior intensidade do pico cristalográfico $100 \%$. Pode-se atribuir que o aumento da concentração do dopante na matriz promova um aumento na densidade de defeitos que podem ser do tipo vacâncias de oxigênio. Assim sendo, essa maior densidade de defeitos provocaria a supressão da luminescência da amostra $\mathrm{CeO}_{2}$ dopada com $5 \%$ de magnésio. Para melhor estudar o comportamento das emissões fotoluminescência observadas, os espectros de emissão fotoluminescente foram decompostos usando uma função Gaussiana. Esta decomposição foi realizada com o programa PeakFit (versão 4.05). A Fig. 6 ilustra o ajuste realizado para as curvas de fotoluminescência das amostras de $\mathrm{CeO}_{2}$ com diferentes dopagens obtidas em 800 ${ }^{\circ} \mathrm{C}$, para avaliar o efeito da dopagem sobre o comportamento fotoluminescente. A Fig. 7 ilustra o ajuste realizado para as curvas de fotoluminescência das amostras de $\mathrm{CeO}_{2}$ dopadas 

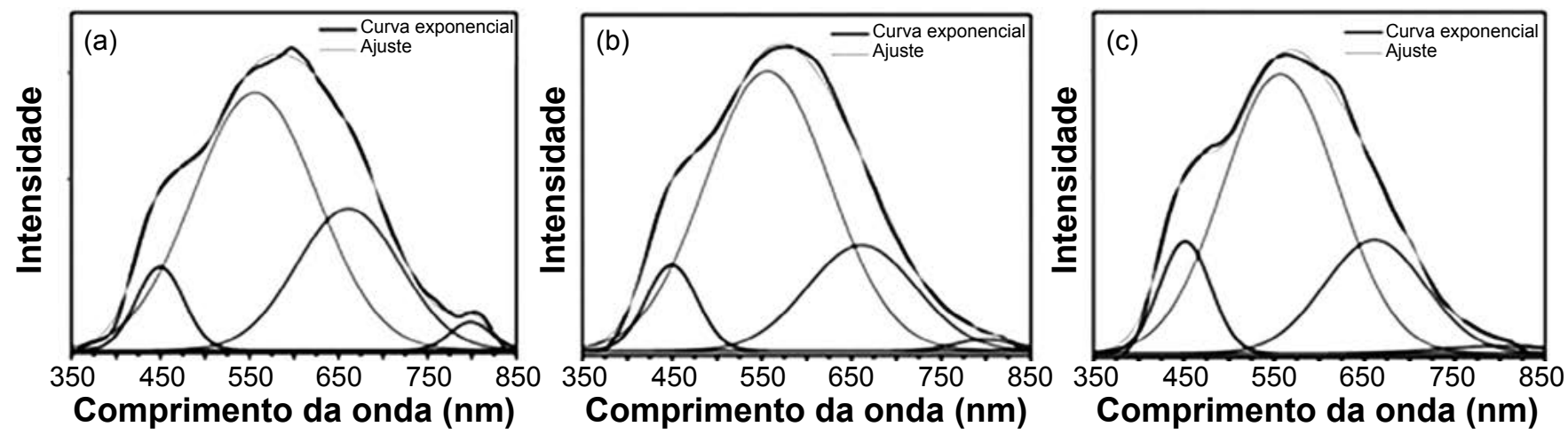

Figura 6: Decomposição dos espectros de emissão fotoluminescência da amostra de $\mathrm{CeO}_{2}$ dopada com (a) $1 \%$, (b) $2 \%$ e (c) $5 \%$ de magnésio obtida em $800{ }^{\circ} \mathrm{C}$.

[Figure 6: Decomposition of the photoluminescence emission spectra of the sample $\mathrm{CeO}_{2}$ doped with (a) $1 \%$, (b) $2 \%$ and (c) $5 \%$ magnesium at $\left.800^{\circ} \mathrm{C}.\right]$

com $2 \%$ magnésio obtidas em $500,600,700$ e $800{ }^{\circ} \mathrm{C}$, para avaliar o efeito da temperatura sobre o comportamento fotoluminescente. A função Gaussiana foi utilizada com sucesso para ajustar os picos de fotoluminescência e os parâmetros de ajuste, incluindo a posição dos picos e das áreas percentuais correspondentes, Tabelas I, para as amostras com diferentes dopagens obtidas em $800{ }^{\circ} \mathrm{C}$, e na Tabela II para as amostras dopadas com $2 \%$ de magnésio obtidas em diferentes temperaturas.

Neste primeiro caso, a decomposição pode ser interpretada como sendo composta por quatro componentes. Essas componentes são aqui chamadas de azul (máximo de intensidade fotoluminescente menor que $500 \mathrm{~nm}$ ), componente verde (máximo menor que $590 \mathrm{~nm}$ ), componente vermelha (máximo menor que $740 \mathrm{~nm}$ ) e componente infravermelha (valores acima de $740 \mathrm{~nm}$ ). Cada cor representa um conjunto de transições eletrônicas diferentes e estão relacionadas a um arranjo estrutural específico.

Para todas as amostras obtidas em $800{ }^{\circ} \mathrm{C}$ a contribuição máxima de emissão fotoluminescente ocorre na faixa de luz verde e com o aumento da porcentagem de dopante é observado um ligeiro aumento da contribuição na faixa de luz azul do espectro eletromagnético.

A Fig. 7 ilustra o ajuste realizado para as curvas de fotoluminescência das amostras de $\mathrm{CeO}_{2}$ dopadas com $2 \%$ de magnésio obtidas em $500,600,700$ e $800^{\circ} \mathrm{C}$, para avaliar o efeito da temperatura sobre o comportamento fotoluminescência.
Neste segundo caso a decomposição pode ser interpretada como sendo composta por três componentes. Pode-se observar que para a amostra obtida em $500^{\circ} \mathrm{C}$ a contribuição máxima de emissão fotoluminescente ocorre na faixa de luz azul e com o aumento da temperatura de tratamento térmico a contribuição máxima passa a ser na faixa de luz vermelha. Como a fotoluminescência está associada à ordem-desordem estrutural do composto, mesmo pequenas alterações estruturais podem provocar mudanças nas interações entre os estados eletrônicos, levando à formação de níveis que podem estar localizados mais próximos da banda de valência ou da banda de condução, acarretando diferentes comportamentos da fotoluminescência.

Com a finalidade de detectar e compreender o efeito das distorções estruturais na distribuição dos estados eletrônicos do material, foram realizadas análises de absorção de luz entre $200 \mathrm{a} 800 \mathrm{~nm}$, correspondente às regiões do ultravioleta e do visível. Este tipo de medida permite estimar o valor do band gap ótico de cerâmicas por medidas de refletância difusa total. Os espectros de absorção da Fig. 8 referem-se ao $\mathrm{CeO}_{2}$ dopado com $2 \%$ magnésio tratado termicamente em mais baixa temperatura, $500^{\circ} \mathrm{C}$, o qual possui um band gap de $2,89 \mathrm{eV}$, e a $700{ }^{\circ} \mathrm{C}$, em que o band gap é $3,06 \mathrm{eV}$. Os resultados obtidos pelos espectros e pelos cálculos de band gap indicam que ocorre um aumento nesses valores com o aumento da temperatura de tratamento, e isto está associado à redução dos níveis intermediários no gap óptico deste

Tabela I - Valores de centro de emissão e área percentual de picos obtidos da deconvolução das curvas de fotoluminescência das amostras de $\mathrm{CeO}_{2}$ obtidas em $800{ }^{\circ} \mathrm{C}$.

[Table I - Emission center and percentage area of the peak deconvolution obtained on the curves from photoluminescence of $\mathrm{CeO}_{2}$ samples obtained at $800^{\circ} \mathrm{C}$.]

\begin{tabular}{ccccccccc}
\hline $\begin{array}{l}\mathrm{Mg} \\
(\%)\end{array}$ & $\begin{array}{c}\text { Pico A } \\
\text { centro } \\
(\mathrm{nm})\end{array}$ & $\begin{array}{c}\text { Area do } \\
\text { Pico A } \\
(\%)\end{array}$ & $\begin{array}{c}\text { Pico B } \\
\text { centro } \\
(\mathrm{nm})\end{array}$ & $\begin{array}{c}\text { Area do } \\
\text { Pico B } \\
(\%)\end{array}$ & $\begin{array}{c}\text { Pico C } \\
\text { centro } \\
(\mathrm{nm})\end{array}$ & $\begin{array}{c}\text { Area do } \\
\text { Pico C } \\
(\%)\end{array}$ & $\begin{array}{c}\text { Pico D } \\
\text { centro } \\
(\mathrm{nm})\end{array}$ & $\begin{array}{c}\text { Area do } \\
\text { Pico D } \\
(\%)\end{array}$ \\
\hline 1 & 449 & 8 & 556 & 61 & 661 & 28 & 798 & 3 \\
2 & 449 & 9 & 556 & 67 & 661 & 23 & 798 & 1 \\
5 & 449 & 12 & 556 & 64 & 661 & 23 & 798 & 1 \\
\hline
\end{tabular}



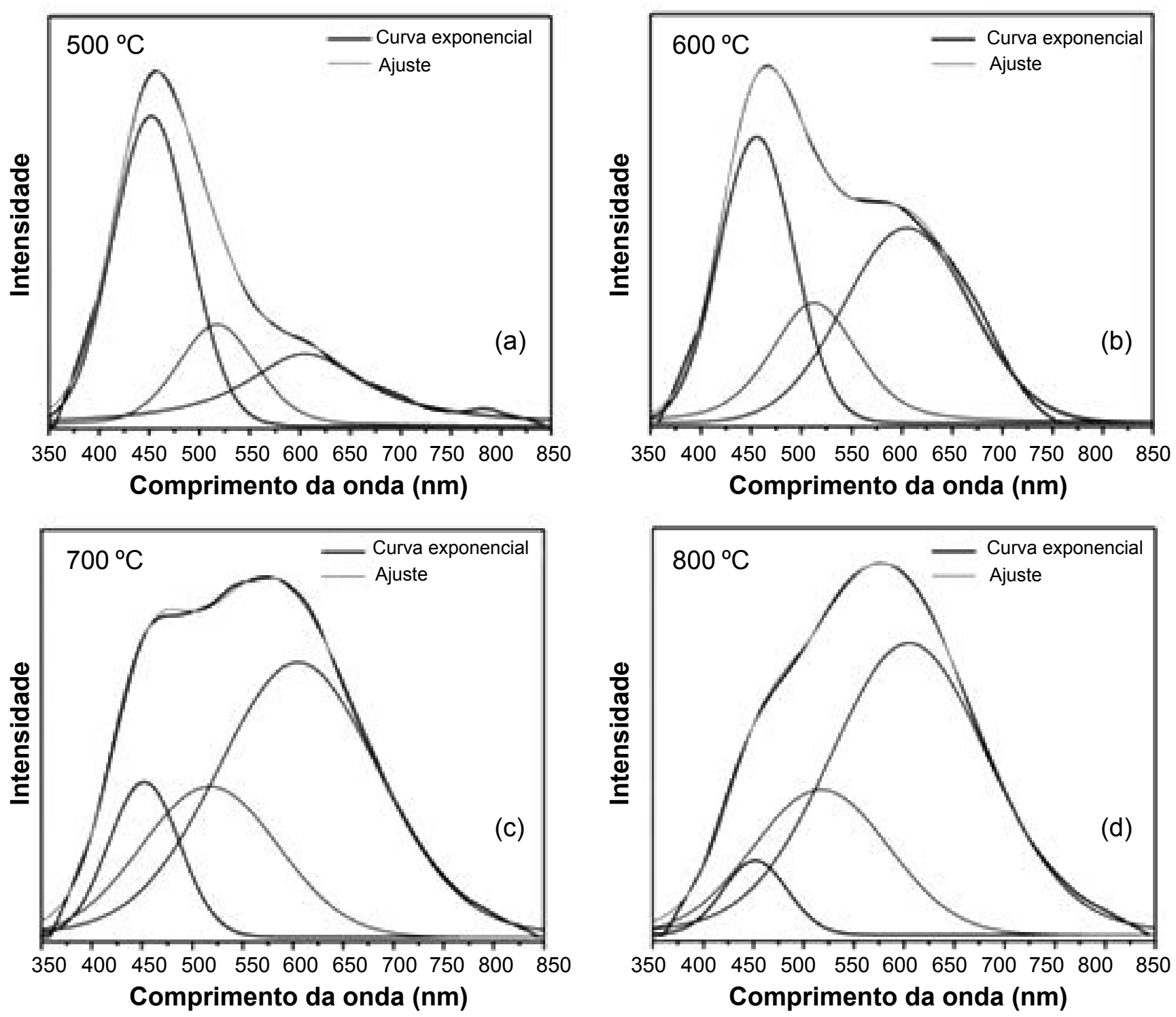

Figura 7: Decomposição dos espectros de emissão fotoluminescente das amostras de $\mathrm{CeO}_{2}$ obtidas em $500,600,700$ e $800{ }^{\circ} \mathrm{C}$.

[Figure 7: Decomposition of the photoluminescence emission spectra of the $\mathrm{CeO}_{2}$ samples obtained at 500, 600,700 and $800{ }^{\circ} \mathrm{C}$.]

Tabela II - Valores de centro de emissão e área percentual de picos obtidos da deconvolução das curvas de fotoluminescência das amostras de $\mathrm{CeO}_{2}$ obtidas em 500, 600, 700 e $800{ }^{\circ} \mathrm{C}$.

[Table II - Emission center and percentage area of the peak deconvolution obtained of the curves from photoluminescence of $\mathrm{CeO}_{2}$ samples obtained at 500,600, 700 and $800^{\circ} \mathrm{C}$.]

\begin{tabular}{ccccccc}
\hline $\begin{array}{c}\mathrm{T} \\
\left({ }^{\circ} \mathrm{C}\right)\end{array}$ & $\begin{array}{c}\text { Pico A } \\
\text { centro } \\
(\mathrm{nm})\end{array}$ & $\begin{array}{c}\text { Area do } \\
\text { Pico A } \\
(\%)\end{array}$ & $\begin{array}{c}\text { Pico B } \\
\text { centro } \\
(\mathrm{nm})\end{array}$ & $\begin{array}{c}\text { Area do } \\
\text { Pico B } \\
(\%)\end{array}$ & $\begin{array}{c}\text { Pico C } \\
\text { centro } \\
(\mathrm{nm})\end{array}$ & $\begin{array}{c}\text { Area do } \\
\text { Pico C } \\
(\%)\end{array}$ \\
\hline 500 & 451 & 52 & 517 & 19 & 605 & 29 \\
600 & 451 & 37 & 517 & 21 & 605 & 42 \\
700 & 451 & 14 & 517 & 26 & 605 & 60 \\
800 & 451 & 6 & 517 & 27 & 605 & 67 \\
\hline
\end{tabular}

material devido ao aumento da organização estrutural. Estes valores estão bem próximos do reportado para este mesmo material, $2,94 \mathrm{eV}$ [33].

Análises de microscopia eletrônica de varredura foram realizadas com o intuito de monitorar o grau de aglomeração, forma e tamanho das partículas, bem como o efeito da temperatura de tratamento térmico para os pós cerâmicos de $\mathrm{CeO}_{2}$ dopado com magnésio. A Fig. 9 apresenta as 

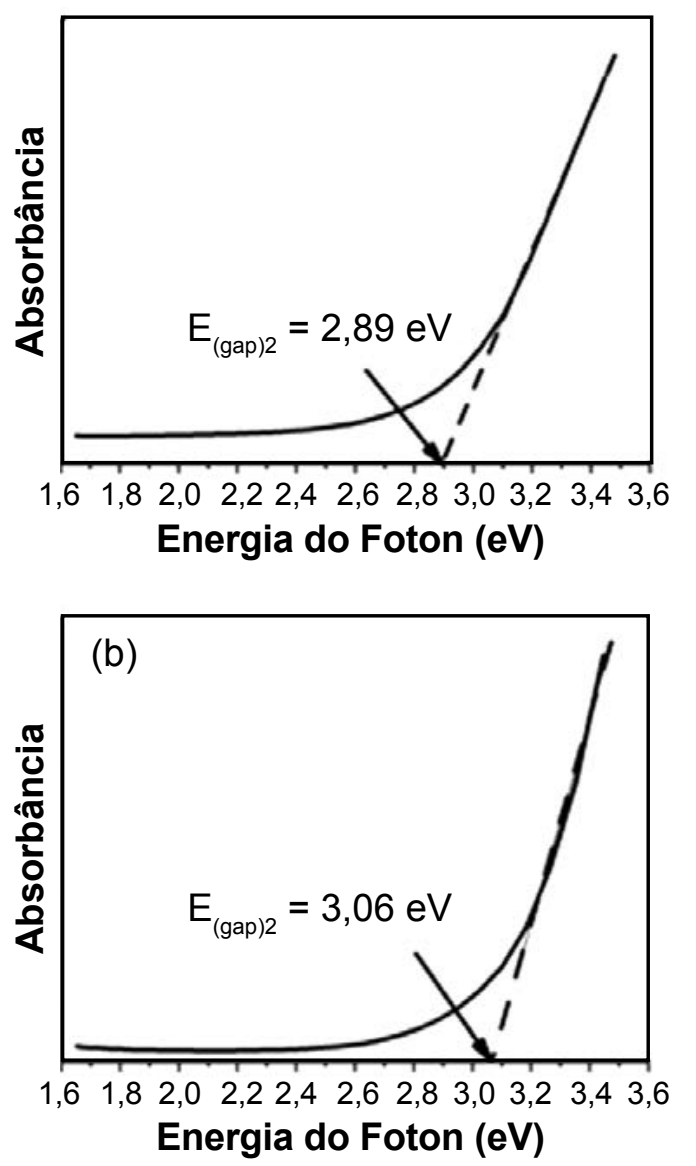

Figura 8: Espectros de absorbância para os pós das amostras $\mathrm{CeO}_{2}$ dopados com $2 \%$ magnésio tratados termicamente de (a) 500 e (b) $800{ }^{\circ} \mathrm{C}$.

[Figure 8: Absorbance spectra of powders samples of $\mathrm{CeO}_{2}$ doped with $2 \%$ magnesium heat-treated of (a) 500 and (b) $800{ }^{\circ} \mathrm{C}$.]

(a)

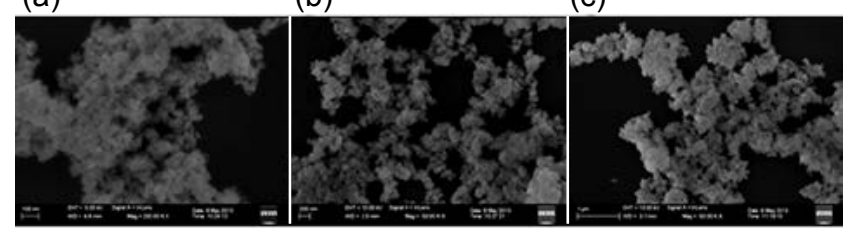

Figura 9: Micrografias obtidas por microscopia eletrônica de varredura dos pós de $\mathrm{CeO}_{2}$ dopado com (a) $1 \%$, (b) $2 \%$ e (c) $5 \%$ de magnésio, tratados termicamente a $800{ }^{\circ} \mathrm{C}$.

[Figure 9: SEM micrographs of the $\mathrm{CeO}_{2}$ powders doped with (a) $1 \%$, (b) $2 \%$ and (c) $5 \%$ magnesium, thermally treated at $\left.800{ }^{\circ} \mathrm{C}.\right]$

fotomicrografias obtidas para os pós tratados termicamente a $800{ }^{\circ} \mathrm{C}$. As partículas possuem formas bastante irregulares e apresentam um elevado grau de aglomeração. Este problema poderá ser controlado possivelmente por um processo de moagem após as etapas de tratamento térmico, em que é possível obter um sistema menos aglomerado e com menor tamanho de partícula.

\section{CONCLUSÕES}

Os pós de estruturas ordenadas de $\mathrm{CeO}_{2}$ dopado com magnésio foram obtidos com sucesso pelo método dos precursores poliméricos. As amostras de $\mathrm{CeO}_{2}$ dopada com $2 \%$ de magnésio apresentaram máxima intensidade de emissão fotoluminescente, enquanto que nas amostras de $\mathrm{CeO}_{2}$ dopada com $5 \%$ de magnésio houve uma diminuição nessa intensidade. Esse fenômeno pode ser atribuído ao "quenching" (supressão) de concentração. Observou-se por difração de raios $\mathrm{X}$ que a amostra $\mathrm{CeO}_{2}$ dopada com $2 \%$ de magnésio apresentou uma maior cristalinidade, ou seja, organização estrutural a longa distância. Atribuiu-se que com o aumento da concentração do dopante na matriz promoveuse um aumento na densidade de defeitos, que podem ser do tipo vacâncias de oxigênio. Essa maior densidade de defeitos provocou a supressão da luminescência da amostra de $\mathrm{CeO}_{2}$ dopada com 5\% de magnésio.

\section{AGRADECIMENTOS}

À FAPESP (CEPID Proc. 2013/07296-2). Um dos autores agradece ao CNPq (Proc. 161641/2011-3) pela bolsa PDJ.

\section{REFERÊNCIAS}

[1] S. Gnanam, V. Rajendran, J. Sol-Gel Sci. Technol. 58 (2011) 62-69.

[2] S. K. Kim, S. Lee, U. Paik, T. Katoh, J. G. Park, J. Mater. Res. 18 (2003) 2163-2169.

[3] S. T. Aruna, S. Ghosh, K. C. Patil, Int. J. Inorg. Mater. 3 (2001) 387-392.

[4] B. Feng, C. Y. Wang, B. Zhu, Electrochem. Solid State Lett. 9 (2006) A80-A81.

[5] T. S. Zhang, J. Ma, L. B. Kong, S. H. Chan, J. A. Kilner, Solid State Ionics 170 (2004) 209-217.

[6] P. Jasinski, T. Suzuki, H. U. Anderson, Sensors Actuators B, Chemical 95 (2003) 73-77.

[7] S. Fujihara, M. Oikawa, J. Appl. Phys. 95 (2004) 80028006.

[8] Z. Wang, Z. Quan, J. Lin, Inorg. Chem. 46 (2007) $5237-$ 5242 .

[9] T. Karaca, T. G. Altıncekic, M. F. Oksuzomer, Ceram. Int. 36 (2010) 1101-1107.

[10] E. C. C. Souza, H. F. Brito, E. N. S. Muccillo, J. Alloys Compnd. 491 (2010) 460-464.

[11] E. C. C. Souza, E. N. S. Muccillo, J. Alloys Compnd. 473 (2009) 560-566.

[12] M. Llusar, L. Vitaskova, P. Sulcova, M. A. Tena, J. A. Badenes, G. Monros, J. Eur. Ceram. Soc. 30 (2010) 37-52.

[13] L. Li, H. K. Yang, B. K. Moon, Z. Fu, C. Guo, J. H. Jeong, S. S. Yi, K. Jang, H. S. Lee, J. Phys. Chem. C113 (2009) 610-617.

[14] A. A. Ansari, A. Kaushik, J. Semicond. 31 (2010) 033001-1-4.

[15] P. S. Anjana, T. Joseph, M. T. Sebastian, J. Alloys Compnd. 490 (2010) 208-213.

[16] Y. P. Fu, Ceram. Int. 35 (2009) 653-659.

[17] S. Xinyuan, G. Mu, Z. Min, H. Shiming, J. Rare Earths 28 (2010) 340-344. 
[18] L. Zeng, D. Chen, L. Cui, F. Huang, Y. Wang, Scripta Mater. 63 (2010) 661-664.

[19] S. Babu, A. Schulte, S. Seal, Appl. Phys. Lett. 92 (2008) 123112 .

[20] G. Blasse, B. C. Grabmaier, Luminescent Materials, 1르 Ed., Springer, Berlin, Alemanha (1994) 234.

[21] L. T. Canham, Appl. Phys. Lett. 57 (1990) 1046.

[22] A. H. Morhed, M. E. Moussa, S. M. Bedair, R. Leonard, S. X. Liu, N. A. El-Masry, Appl. Phys. Lett. 75 (1997) 2389.

[23] S.-H. Yu, H. Colfen, A. Fischer, Colloids Surfaces A243 (2004) 49-52.

[24] S. Sathyamurthy, K. J. Leonard, R. T. Dabestani, M. P. Paranthaman, Nanotechnol. 16 (2005) 1960-1964.

[25] M. Y. Chen, X. T. Zu, X. Xiang, H. L. Zhang, Physica B 389 (2007) 263-268.

[26] F. Gao, G. H. Li, J. H. Zhang, F. G. Qin, Z. Y. Yao, Z. K. Liu, Z. G. Wang, L. Y. Lin, Chinese Phys. Lett. 18 (2001) 443-444.

[27] S. Phokaa, P. Laokula, E. Swatsitanga, V. Promarakb,
S. Seraphinc, S. Maensiria, Mater. Chem. Phys. 115 (2009) 423-428.

[28] F. Meng, L. Wanga, J. Cui, J. Alloys Compnd. 556 (2013) 102-108.

[29] E. Yu. Pikalova, A. A. Murashkina, V. I. Maragou, A. K. Demin, V. N. Strekalovsky, P. E. Tsiakaras, Int. J. Hydrogen Energy 36 (2011) 6175-6183.

[30] I. Kosacki, T. Suzuki, U. H. Anderson, P. Colomban, Solid State Ionics 149 (2002) 99-105.

[31] V. M. Longo, L. S. Cavalcante, A. T. de Figueiredo, L. P. S. Santos, E. Longo, J. A. Varela, J. R. Sambrano, C. A. Paskocimas, F. S. De Vicente, A. C. Hernandes, Appl. Phys. Lett. 90 (2007) 091906-8.

[32] M. L. Moreira, M. F. C. Gurgel, G. P. Mambrini, E. R. Leite, P. S. Pizani, J. A. Varela, E. Longo, J. Phys. Chem. A112 (2008) 8938-8942.

[33] J. M. Coronado, A. J. Maira, A. Martínez-Arias, J. C. Conesa, J. Soria, J. Photochem. Photobiol. A150 (2002) 213-224.

(Rec. 28/10/2013, Ac. 28/01/2014) 